\title{
Psicoeducação para familiares de portadores de transtorno mental grave em um hospital geral
}

\author{
Psychoeducation for families of serious mental disorders in a general hospital \\ Psicoeducación para familias con trastornos mentales graves en un hospital general
}

\begin{abstract}
RESUMO
Objetivo: relatar a experiência do grupo de "psicoeducação" aos familiares de pacientes com transtorno mental grave internados em um hospital geral do sul do Brasil. Método: relato de experiência sobre o grupo de "psicoeducação" para familiares de pacientes com de transtorno mental grave internados em uma Unidade Psiquiátrica em um hospital geral do Sul do Brasil. As vivências relatadas por duas enfermeiras ocorreram no período de novembro de 2019 a maio de 2020. Resultados: o grupo de "psicoeducação" é um recurso valioso na intervenção com as famílias, mostrando-se ser vantajoso ao ser implementado em um hospital geral, trazendo enfoque ao ato de cuidar dos processos saúde-doença das famílias. Conclusão: Com o grupo de "psicoeducação" é possível implementar estratégias de promoção, prevenção e cuidado de enfermagem, não apenas para o indivíduo, mas também para a família.
\end{abstract}

DESCRITORES: Educação em Saúde; Família; Transtorno Mental

\section{ABSTRACT}

Objective: to report the experience of using the "psychoeducation" group to family members of patients with severe mental disorders admitted to a general hospital in southern Brazil. Method: experience report on the "psychoeducation" group for family members of patients with severe mental disorder hospitalized in a Psychiatric Unit of a general hospital in southern Brazil. The experiences reported by two nurses took place from November 2019 to May 2020. Results: the "psychoeducation" group is a valuable resource in intervention with families, proving to be advantageous when implemented in a general hospital, focusing on the act of caring for the health-disease processes of families. Conclusion: With the "psychoeducation" group it is possible to implement strategies for promotion, prevention and nursing care, not only for the individual, but also for the family.

DESCRIPTORS: Health Education; Family; Mental Disorder

\section{RESUMEN}

Objetivo: reportar la experiencia de uso del grupo de "psicoeducación" a familiares de pacientes con trastornos mentales graves ingresados en un hospital general del sur de Brasil. Método: relato de experiencia del grupo de "psicoeducación" para familiares de pacientes con trastorno mental severo hospitalizados en una Unidad Psiquiátrica de un hospital general del sur de Brasil. As vivências relatadas por duas enfemeiras ocorreram no período de novembro de 2019 a maio de 2020. Resultados: el grupo de "psicoeducación" es un recurso valioso en la intervención con las familias, resultando ventajoso cuando se implementa en un hospital general, enfocándose en el acto de cuidar los procesos salud-enfermedad de las familias. Conclusión: Con el grupo de "psicoeducación" es posible implementar estrategias de promoción, prevención y cuidado de enfermería, no solo para el individuo, sino también para la familia.

DESCRIPTORES: Educación en Salud; Familia; Transtorno mental

RECEBIDO EM: 08/06/21 APROVADO EM: 14/06/21

\section{Vanessa Menegalli}

Especialista em Saúde da Família, Enfermeira do Hospital de Clínicas de Porto Alegre, Porto Alegre, Rio Grande do Sul Orcid: 000-0001-7322-2753.

\section{Francine Morais da Silva}

Enfermeira, Mestra em Enfermagem, Universidade Federal do Rio Grande do Sul/Escola de Enfermagem, Porto Alegre, Rio Grande do Sul.

Orcid: 0000-002-9226-7663.

\section{Aline Oliveira}

Enfermeira do Hospital de Clínicas de Porto Alegre, Especialista em Saúde da Família, Porto Alegre, Rio Grande do Sul

Orcid: 0000-0002-9873-8446. 


\section{INTRODUÇÃO}

A internação de pessoas portadoras de Transtornos Mental Grave no Brasil remonta à metade do Século XIX. Desde então, a atenção destinada ao tratamento de pacientes portadores de Transtornos Mental Grave foi por longos anos sinônimo de internação em hospitais psiquiátricos especializados. Com a proclamação da Constituição, em 1988, cria-se o Sistema Único de Saúde (SUS) e são estabelecidas as condições institucionais para a implantação de novas políticas de saúde, entre as quais a de saúde mental, denominada Reforma Psiquiátrica1.

A Reforma Psiquiátrica possibilitou inúmeras transformações na assistência à saúde mental, sendo que o tratamento terapêutico fez parte dessa transformação. Sua prática vem se fortalecendo dentro do novo modelo assistencial, mostrando que veio para preencher as lacunas deixadas pelos tratamentos psiquiátricos tradicionais, onde o alvo principal era o paciente e sua patologia. Na maioria das vezes a internação hospitalar ocorria por longos períodos, excluindo o portador de Transtorno Mental Grave da convivência familiar, afastando-os da responsabilidade de participar do cuidado ao mesmo2.

A indicação de internação psiquiátrica pode ser um episódio de intenso sofrimento para o paciente e seus familiares, visto que essa indicação é consequência do esgotamento de todos os recursos terapêuticos extra-hospitalares, bem como, quando não há condições mínimas de proteção para o paciente e sua família3.

O Transtorno Mental Grave é uma doença crônica, atingindo não só a pessoa individualmente, mas todas de seu convívio, sendo de extrema importância a existência de serviços que contribuam com o processo coletivo familiar da convivência cotidiana do processo doença/doente mental. Nos serviços de atenção à saúde mental os familiares normalmente se apresentam como meros "informantes" das alterações do paciente, não sendo sensibilizados e orientados para se tornarem importantes atores no processo4.
A Reforma

Psiquiátrica

possibilitou

inúmeras

transformações na

assistência à saúde

mental, sendo

que o tratamento

terapêutico

fez parte dessa

transformação.

Sua prática vem

se fortalecendo

dentro do novo

modelo assistencial,

mostrando que veio

para preencher as

lacunas deixadas

pelos tratamentos

psiquiátricos

tradicionais, onde

o alvo principal era

o paciente e sua

patologia
Para os pacientes, com Esquizofrenia e Transtorno Afetivo Bipolar, a internação psiquiátrica pode ser vista como espaço potencializador do tratamento, já que as unidades de internação reúnem diversos profissionais, famílias e o indivíduo no seu momento de crise 5 .

Nesse contexto, cabe destacar que cerca de 23 milhôes de pessoas são portadoras de esquizofrenia no mundo. Em relação ao Brasil, existem cerca de dois milhões de brasileiros portadores do referido distúrbio. Já no que tange o Transtorno Afetivo Bipolar acomete cerca de $4 \%$ da população brasileira4-5.

A família tem um papel muito importante em todo o tratamento terapêutico, desde a adesão do doente ao tratamento medicamentoso, bem como a todos os processos implantados visando a melhoria da qualidade de vida do portador de Transtorno Mental Grave5.

O apoio prestado no convívio familiar fortalece os laços afetivos e auxiliam no entendimento da patologia melhorando os serviços dos profissionais de saúde nas diversas instituições prestadoras da assistência ao doente mental. Os encontros realizados com familiares de portadores de transtorno mental ajudam a compreender a convivência familiar e suas dificuldades para lidar com suas limitações5.

A Psicoeducação é uma abordagem terapêutica na qual as informações específicas sobre a doença são fornecidas aos familiares do paciente, é um processo na qual um facilitador (profissional de saúde) detalha e resume informações científicas e relevantes para responder perguntas. Sobre a origem, evolução e abordagem de uma doença a partir de seu ambiente, situação que também implica aconselhamento e treinamento em técnicas para lidar com o problema6.

Um dos principais objetivos da Psicoeducação é a adesão à medicação. Ela visa dar aos familiares informações sobre a natureza e o tratamento do Transtorno Mental Grave, provendo ensinamentos teóricos e práticos para que o paciente possa compreender e lidar melhor com a sua doença. A Psicoeducação pode abordar outros tópicos como as intervenções psicoeducacionais, a identi- 
ficação precoce dos sintomas prodrômicos, a coibição de drogas de abuso e o manejo de situações provocadoras do estresse e da ansiedade. Quando combinada com tratamento farmacológico, a psicoeducação ajuda a melhorar a aderência ao tratamento7. A Psicoeducação é, também, útil no auxílio de familiares e pessoas próximas do paciente8.

Os benefícios da participação do familiar no processo de Psicoeducação para a adesão ao tratamento medicamentoso, bem como nos resultados terapêuticos obtidos em pacientes com Transtorno Afetivo Bipolar e Esquizofrenia são indiscutíveis. Além disso, algumas investigações mostram que familiares de pacientes com transtorno afetivo bipolar e esquizofrenia possuem índices de estresse muito elevados 9 .

A psicoeducação auxilia paciente e familiar a lidar com a estigmatização, favorece a adesão ao tratamento, ensinar o paciente e a família a identificar os sinais prodrômicos precoces além de promover hábitos saudáveis 10.

Nesse sentido, o presente estudo tem como objetivo relatar a experiência da utilização da técnica de intervenção "psicoeducação" aos familiares de pacientes com transtorno mental grave internados em um hospital geral do sul do Brasil.

\section{MÉTODO}

Trata-se de um relato de experiência de natureza descritiva/reflexiva, do tipo relato de experiência, onde buscou-se descrever a experiência do grupo de "psicoeducação" para familiares de pacientes com transtorno mental grave internados em um hospital geral do sul do Brasil, realizado no período de novembro de 2019 a maio de 2020.

A Unidade de Internação Psiquiátrica de um Hospital Geral do Sul do Brasil foi inaugurada em 1988, configurando-se como um serviço que dispóe de 36 leitos, destes 26 conveniados com o SUS e 10 leitos privativos. A porta de entrada dos pacientes do SUS é regulada pela Central de Leitos do Estado do Rio Grande do Sul. No ano de 2014, totalizaram 435 internações pelo Sistema Único de Saúde.
Os benefícios da participação do familiar no processo de Psicoeducação

para a adesão

ao tratamento

medicamentoso,

bem como

nos resultados

terapêuticos obtidos

em pacientes

com Transtorno

Afetivo Bipolar e

Esquizofrenia são

indiscutíveis. Além

disso, algumas

investigaçôes

mostram que

familiares de

pacientes com

transtorno

afetivo bipolar

e esquizofrenia

possuem índices

de estresse muito

elevados
A equipe multidisciplinar da Unidade de Internação Psiquiátrica é composta por médicos, assistente social, farmacêutica, psicóloga, nutricionista e educador físico, residentes multiprofissionais (médicos, enfermeiro, psicólogo e educador físico), enfermeiros e técnicos de enfermagem.

A referida Unidade de Internação Psiquiátrica disponibiliza semanalmente um grupo de família para os familiares de pacientes portadores de Transtorno Mental Grave (Esquizofrenia e Transtorno Afetivo Bipolar) com intuito de acolher demandas dos familiares, escuta ativa, esclarecimento de dúvidas a respeito das patologias psiquiátricas. O grupo de "psicoeducação" é coordenado por duas enfermeiras (profissionais de nível superior). Tem por base a intervenção psicossocial, eficaz para adesão ao tratamento psicofarmacológico.

Como critérios para participação no grupo foram: a) familiares maiores de 18 anos; b) familiares de pacientes com diagnóstico clínico de Esquizofrenia ou Transtorno Afetivo Bipolar; c) ser familiar de pacientes internados na Unidade de Internação Psiquiátrica de um Hospital do Sul do Brasil. Como critérios de exclusão: a) familiares sem disponibilidade e/ou interesse em participar do Grupo de Psicoeducação.

No grupo foram trabalhadas temáticas e metodologias diversas, como: informação sobre os altos índices de recorrência associados à doença e à sua condição crônica; informação sobre os fatores de desencadeamento e um treinamento pessoal para auxiliar os pacientes a identificarem os seus próprios; informação sobre os agentes psicofarmacológicos, suas vantagens e seus potenciais efeitos colaterais; treinamento na detecção precoce dos sintomas prodrômicos; treinamento sobre o manejo dos sintomas; informação sobre os riscos associados ao uso de drogas ilícitas, café e álcool; promoção de hábitos saudáveis; treinamento em gerenciamento de estresse; informação concreta sobre alguns assuntos como gravidez e transtornos bipolares e risco de suicídio; lidar com o estigma e outros problemas sociais relacionados à doença que os pacientes bipolares não podem discutir facilmente com seus amigos "saudáveis". 
Ao final de cada encontro as duas enfermeiras reuniam-se para registro das atividades em prontuário, realizavam avaliação do grupo e direcionamentos das demandas. Por fim, o registro das informações que compõe e instrumentalizam o estudo parte de anotações realizadas em agenda de trabalho própria para o grupo.

\section{RESULTADOS E DISCUSSÃO}

Os resultados foram estruturados e organizados a partir da observação do grupo semanal de "Psicoeducação" para familiares de pacientes com transtorno mental grave internados em um hospital geral do sul do Brasil realizado por duas enfermeiras da unidade de internação psiquiátrica em um hospital geral do sul do Brasil. O relato consiste em apresentar as percepções obtidas por enfermeiras em uma unidade de Internação Psiquiátrica de um hospital geral do sul do Brasil.

A experiência do Grupo de "Psicoeducação" foi extremamente rica para os familiares. Através do entendimento da doença, bem como esclarecimento de dúvidas, os pacientes se sentiam melhor compreendidos o que possibilitava uma maior consciência, motivação e esperança para a realização de um tratamento eficaz.

A psicoeducação em grupos possibilita novos conhecimentos sobre as suas demandas e também ajuda diretamente no vínculo entre profissional e familiar, facilitando o entendimento de seus problemas, doenças, ao esclarecer dúvidas e mostrando que os familiares/acompanhantes são ativos na terapia10.

A Psicoeducação no cenário familiar gera processos de empoderamento e concede os recursos necessários a seus membros para que eles possam desenvolver comportamentos saudáveis. Como se pode inferir, a responsabilidade familiar é decisiva no tratamento, uma vez que é o primeiro sistema encarregado de influenciar os hábitos e costumes das pessoas. Com tudo isso, a promoção da saúde, através da Psicoeducação, é necessária, pois atua de forma sistemática e participativa com os familiares, trazendo benefícios diretos à sua saúde9.
A psicoeducação

demonstrou sua eficácia na profilaxia de todos os tipos de recidivas aos $2 \mathrm{e}$ 5 anos, reduzindo assim a duração dos episódios, hospitalizações

e problemas de adesão. Aliviar a carga do cuidador e permitir que as famílias continuem exercendo sua função de cuidador, a fim de impedir a institucionalização
Os participantes que frequentavam regularmente o grupo de "Psicoeducação" estavam mais satisfeitos com o seu papel, aprenderam novas formas de lidar com os conflitos familiares e apresentaram maior autoestima após a intervenção.

A aplicação do grupo psicoeducacional esteve associada a uma queda significativa da sobrecarga, que reduziu os efeitos sociais e psicológicos do cuidador após a intervenção, como: pouco tempo livre, dificuldades no trabalho, sentimentos de angústia, raiva e medo.

Os pacientes cujos familiares participaram de uma intervenção psicoeducacional sistemática, adaptada às particularidades dos traços de personalidade, apresentaram melhor expectativa de autonomia e recuperação, portanto, a percepção de apoio com a família melhorou e a insatisfação diminuiu.

A psicoeducação demonstrou sua eficácia na profilaxia de todos os tipos de recidivas aos 2 e 5 anos, reduzindo assim a duração dos episódios, hospitalizações e problemas de adesão. Aliviar a carga do cuidador e permitir que as famílias continuem exercendo sua função de cuidador, a fim de impedir a institucionalização9.

Além disso, os grupos de apoio multifamiliares contam com diversos fatores terapêuticos, como instilação de esperança, solidariedade, desenvolvimento de técnicas de socialização, comportamento imitativo, libertação de emoções e aprendizagem interpessoal11.

Um estudo avaliou os resultados do oferecimento de um grupo de apoio e encontrou como aspectos positivos para os participantes a ampliação dos recursos sociais, maior nível de conhecimento sobre as doenças, aumento da capacidade de enfrentamento das situações da vida, melhora na autoconfiança, diminuição do medo e da ambiguidade, alívio emocional e redução da desesperança9-12

Em relação à intervenção psicoeducacional, cabe destacar que esta envolve um conjunto de abordagens que tem a função de fornecer conhecimento aos doentes e seus familiares sobre o transtorno mental, novas formas de lidar com ele, formas de tratamento, as necessidades do paciente, a 
redução do estresse originado das relações interpessoais, a adequação do grau de expectativas e exigências em relação ao doente e a prevenção de recorrências. Esse tipo de abordagem pode ser realizado juntamente com os grupos de apoio ou individualmente com cada família10.

Além disso, deve envolver a participação de uma equipe multidisciplinar para ampliar as informações e sanar as dúvidas de todos os âmbitos da doença. A efetividade dessa intervenção é observada na diminuição significativa dos episódios de recaídas e internaçôes e na melhora do funcionamento psicossocial do paciente, além do bem-estar geral da família8.

\section{CONCLUSÃO}

A Psicoeducação pode promover aos familiares/acompanhantes informações sobre o transtorno e com o objetivo de torná-lo colaborador ativo no tratamento, tornando o processo terapêutico mais efetivo.

Salienta-se a importância da psicoeducação em grupo como técnica para engajar os familiares no tratamento bem como ampliar o sentimento de mútua ajuda entre eles. A partir desse trabalho, pode-se verificar importância do grupo psicoeducativo para familiares. Isso ocorre com propósito de acrescentar informaçôes importantes a respeito da psicopatologia, e também pelo clima de integração e mútua-ajuda presente no contexto.
A psicoeducação aplicada pela enfermagem favorece uma aliança terapêutica, também reconhece o ambiente em que a família se desenvolve, ajuda a aumentar as habilidades e comportamentos necessários para promover a saúde individual e coletivo.

Enquanto limitação, este estudo foi realizado em apenas um serviço especializado no atendimento a pacientes com transtorno mental grave em um dado contexto assistencial no sul do Brasil, cabendo à ampliação da realização de estudos semelhantes, em outros serviços voltados à assistência em saúde mental com diferentes características epidemiológicas e de constituição de rede assistencial social e em saúde.

\section{REFERÊNCIAS}

1 Tavares JN. O cuidado psicossocial no campo da saúde mental infantojuvenil: desconstruindo saberes e reinventando saúde. Saúde debate. 2020;44(127):1176-1188.

2 Silva JS, Ribeiro HKP, Fernandes MA, Rocha DM. O cuidar de enfermagem em saúde mental na perspectiva da reforma psiquiátrica. Enfermagem em Foco. 2020;11(1);170-175.

3 Fatureto MLP, Paula-Ravagnani GS, Guanaes-Lorenzi C. O manejo da internação psiquiátrica compulsória por profissionais de saúde em seu cotidiano. Psicologia \& Sociedade. 2020;32:e190864.

4 Mello RM, Schneider JF. A família e a internação psiquiátrica em hospital geral. Revista Gaúcha de Enfermagem. 2011;32(2):226233.

5 Braga RB, Pegoraro RF. Internação psiquiátrica: o que as famílias pensam sobre isso? Revista Psicologia e Saúde. 2020;12(1):61-73.

6 Carvalho MR, Malagris LEN, Rangé BP. Psicoeducação em terapia cognitivo-comportamental. Novo Hamburgo: Sinopsys; 2019.

7 Silva T, Mello PG, Scheeren P. A psicoeducação no atendimento clínico de depressão refratária. XIV Mostra Científica do Cesuca; novembro 2020. Cachoeirinha, Brasil. Cachoeirinha, Cesuca; 2021.
8 Vilar T, Nogueira MJ, Valentim O, Seabra P. A psicoeducação na adesão terapêutica em utentes com esquizofrenia: uma scoping review. Revista Portuguesa de Enfermagem de Saúde Mental. 2020;(spe):103-108.

9 Zanetti A, Carmo M, Tressoldi L, Vedana K, Giacon B, Martin I. Intervenções familiares em pacientes no primeiro episódio psicótico: evidências da literatura. Revista de Enfermagem UFPE. 2017;11(7):2971-2978.

10 Cuevas-Cancino JJ, Moreno-Pérez NE. Psicoeducación: intervención de enfermería para el cuidado de la familia en su rol de cuidadora. Enfermería universitária;14(3):207-218.

11 Ferreira TPS, Sampaio J, Oliveira IL, Gomes LB. A família no cuidado em saúde mental: desafios para a produção de vidas. Saúde em Debate. 2019;43(121):441-449.

12 Schuewk de Aguiar Russo D, Cautela de Almeida Machado ML. Grupo terapêutico de familiares de crianças com alteração de linguagem. SaudColetiv (Barueri) [Internet]. $29^{\circ}$ de outubro de 2020 [citado $6^{\circ}$ de outubro de 2021];10(52):2264-79. Disponível em: http:// revistas.mpmcomunicacao.com.br/index.php/saudecoletiva/article/ view/548 